\title{
Os benefícios do clampeamento tardio do cordão umbilical
}

\author{
The benefits of late umbilical cord clamping \\ Los beneficios del pinzamiento tardío del cordón umbilical
}

Recebido: 12/09/2021 | Revisado: 17/09/2021 | Aceito: 22/09/2021 | Publicado: 24/09/2021

Gabrielly Laís de Andrade Souza
ORCID: https://orcid.org/0000-0003-2078-9574
Centro Universitário Maurício de Nassau, Brasil
E-mail: gabriellyandrade.enf52@gmail.com
Gabriela de Pontes Siqueira
ORCID: https://orcid.org/0000-0001-9669-7882
Centro Universitário Maurício de Nassau, Brasil
E-mail: gabi.17pontes@ @otmail.com
Anekelly da Silva Oliveira
ORCID: https://orcid.org/0000-0003-2190-8336
Centro Universitário Maurício de Nassau, Brasil
E-mail: gabi.17pontes@ hotmail.com
Manoel Felipe Nunes da Rocha
ORCID: https://orcid.org/0000-0001-5325-888X
Centro Universitário Maurício de Nassau, Brasil
E-mail: manoelfelipeenfermeiro@gmail.com
Monalis Fernanda Soares Silva
ORCID: https://orcid.org/0000-0002-6795-2829
Centro Universitário Maurício de Nassau, Brasil
E-mail: monalisfernanda @gmail.com

\section{Resumo}

Introdução: O American College of Obstetricians and Gynecologists (ACOG), em 2017 recomendou que essa secção do cordão umbilical deveria ser feita de 30 a 60 segundos para os bebês prematuros, que se baseia cientificamente na redução de possíveis complicações relacionadas ao prematuro. Objetivo: Destacar a importância e os benefícios do pinçamento tardio do cordão umbilical. Método: Trata-se de uma revisão integrativa da literatura, realizada nas bases de dados da LILACS; BDENF; MEDLINE; SciELO. Resultados: O corte realizado após 60 segundos apresenta maior transferência sanguínea para o recém-nascido $(\mathrm{RN})$. Porém, ressaltam que alguns profissionais aguardam que o cordão pare de pulsar para realizar o pinçamento, o que resulta em maior eficiência na prática. Conclusão: A realização dessa prática possibilita inúmeros benefícios para o bebê durante o parto e na primeira infância, prevenindo principalmente anemia nessa faixa etária.

Palavras-chave: Clampeamento; Cordão umbilical; Enfermagem obstétrica.

\begin{abstract}
Introduction: The American College of Obstetricians and Gynecologists (ACOG) in 2017 recommended that this section of the umbilical cord should be done in 30 to 60 seconds for premature babies, which is scientifically based on reducing possible complications related to the premature. Objective: To highlight the importance and benefits of late clamping of the umbilical cord. Method: This is an integrative literature review, carried out in LILACS databases; BDENF; MEDLINE; SciELO. Results: The cut performed after 60 seconds shows greater blood transfer to the newborn (NB). However, they point out that some professionals wait for the cord to stop pulsating to perform the clamping, which results in greater efficiency in practice. Conclusion: The performance of this practice allows numerous benefits for the baby during delivery and in early childhood, mainly preventing anemia in this age group.

Keywords: Clamping, Umbilical cord; Obstetric nursing.

\section{Resumen}

Introducción: El Colegio Americano de Obstetras y Ginecólogos (ACOG) en 2017 recomendó que esta sección del cordón umbilical se realizara en 30 a 60 segundos para bebés prematuros, lo que se basa científicamente en la reducción de posibles complicaciones relacionadas con el prematuro. Objetivo: Resaltar la importancia y los beneficios del pinzamiento tardío del cordón umbilical. Método: Se trata de una revisión bibliográfica integradora, realizada en bases de datos LILACS; BDENF; MEDLINE; SciELO. Resultados: El corte realizado a los 60 segundos presenta mayor transferencia de sangre al recién nacido (RN). Sin embargo, enfatizan que algunos profesionales esperan a que el cordón deje de latir para realizar el pinzamiento, lo que redunda en una mayor eficiencia en la práctica. Conclusión: La realización de esta práctica aporta innumerables beneficios para el bebé durante el parto y en la primera infancia, principalmente previniendo la anemia en este grupo de edad.
\end{abstract}

Palabras-chave: Sujeción; Cordón umbilical; Enfermería obstétrica. 


\section{Introdução}

No Brasil a assistência ao parto está fortemente marcada pela utilização exagerada de intervenções obstétricas e neonatais, consequentemente causando resultados desfavoráveis no trabalho de parto, o que pode acarretar também a danos tanto a saúde da mãe quanto a do Recém Nascido (RN) (Leal et al., 2019).

Assim, partos definidos como normais e os por cesarianas resultam em assistências diferentes, que estão diretamente relacionadas com o custo benefício de materiais, profissionais e medicamentos, além do que se refere a demanda e ao período de tempo de permanência de cada puérpera nas instituições, tanto públicas quanto privadas (Leal et al., 2019).

As Diretrizes Nacionais de Assistência ao Parto Normal, do Ministério da Saúde (MS), relatam a importância do estímulo do processo fisiológicos e naturais durante o trabalho de parto. Esse fato devido as mudanças que tal processo vem sofrendo nos últimos anos; mudanças essas que favorecem uma melhor prestação da assistência, possibilitando com que ações que preservam a saúde do binômio sejam incentivadas (Brasil, 2017).

Levando em consideração o que foi mencionado, entre outras, uma das práticas que é muito importante é o clampeamento tardio do cordão umbilical, isso por ser uma prática que estimula o processo fisiológico e também tem uma grande efetividade na prestação de benefícios para a puérpera e seu bebê (Brasil, 2017).

Desse modo, esse clampeamento apenas deve ser realizado após o cessamento da pulsação presente no cordão umbilical, priorizando assim o conceito de parto humanizado, que vem priorizar um conforto maior durante o trabalho de parto, que por sinal anteriormente estava sendo deixado de lado $^{2}$.

O Cordão umbilical é um componente exclusivo na formação embrionária que permite a conexão entre o feto e a placenta, o mesmo mede aproximadamente cinquenta centímetros de comprimento e é composto por duas artérias e uma veia, além disso, por um material gelatinoso chamado de geleia de Wharton, uma substância encontrada dentro do cordão que também está presente no humor vítreo do globo ocular e é composta por maior parte de mucopolissacarídeos que contém alguns fibroblastos e macrófagos e é derivada do mesoderma extra-embrionário. O qual tem forte função de troca gasosa fetal e fornecimento de nutrientes e oxigênio para o feto (Malheiros \& Abreu, 2016).

Além do mais, observa-se que desde os primórdios, as práticas constantes em maternidades eram realizadas as secções imediatas do cordão, caracterizado naquela época de: “nasceu, cortou”. Se tornando uma prática comum na década de 70. Assim, alguns pesquisadores dessa época, como Pierre Budin, mencionavam em seus estudos quando deveria ser realizada o clampeamento e questionava sobre a secção precoce. A qual segundo Aristóteles, era uma prática que quando feita cedo era prejudicial, com isto, representou a opinião da comunidade médica, onde Erasmus Darwin abordou que só deveria ser realizada a secção após a pulsação do cordão tenha sido interrompida (Fionda, 2017).

Dessa maneira, a equipe deve protagonizar o espaço da mulher no trabalho de parto, respeitando o processo e as fases do mesmo. Isso acarretará no surgimento de resultados positivos e em uma melhor qualidade da assistência prestada e na saúde tanto do RN, quanto da mãe, minimizando alguns riscos que o parto quando se utiliza de várias atividades intervencionistas pode acarretar. Visto por exemplo, que de acordo com a Organização Mundial da Saúde (OMS) o atraso do clampeamento do cordão umbilical permite a passagem do sangue circulante da placenta para o bebê, no qual cerca de $80 \mathrm{ml}$ a $100 \mathrm{ml}$ de sangue são transferidos, favorecendo o aumento da reserva de ferro com até 50\% (Leal et al., 2019; Vain, 2015).

Levando em consideração o contexto abordado, é descrito pela literatura que a equipe de enfermagem é considerada, na maioria das vezes, os profissionais que estão na linha de frente da assistência a parturiente e ao RN durante todo o trabalho de pato, principalmente se tratando do parto humanizado e da valorização dos aspectos fisiológicos que acompanha tal processo.

Dessa forma, é necessário um profissional qualificado que possa prestar assistência a mulher contemplando os aspectos éticos, humanitários e psicossociais que cercam as mesmas. Diante disso, este trabalho tem como objetivo mencionar a importância do clapeamento tardio e ressaltar os inúmeros benefícios ofertados para a saúde do binômio. 


\section{Metodologia}

O estudo foi realizado através de uma pesquisa bibliográfica do tipo revisão integrativa da literatura, com aspectos descritivos. Nesse caso, optou-se por realizar uma revisão integrativa, por ter uma abordagem metodológica extensa que permite analisar diversos estudos, sendo possível entender e aprender mais sobre o conteúdo já publicado através das revisões.

Os estudos experimentais e não experimentais possibilitam uma visão ampla e completa dos estudos analisados. A análise de dados da literatura teórica e empírica abrange definições e conceitos sobre o estudo, revisão teórica e evidência, como também analisa os problemas metodológicos de um tópico.

Para a elaboração da revisão integrativa foram utilizadas sete etapas: 1) definida pela pergunta norteadora, onde será utilizada para nortear o trabalho, determinar os estudos que serão incluídos, os meios utilizados para identificação e as informações reunidas de cada estudo classificado. 2) escolha da temática, 3) busca e amostragem na literatura, 4) definição da coleta de dados, 5) foi definida pela análise crítica dos estudos incluídos, com rigor metodológico e as características de cada estudo.

Além disso, na 6) foi definido as discursões dos resultados de acordo com as interpretações e síntese dos resultados encontrados, comparado os dados analisados dos artigos com o referencial teórico, 7) apresentação da revisão integrativa de forma clara e objetiva permitindo que o leitor avalie criteriosamente os resultados apresentados. Deverá conter informações oportunas e precisas de acordo com a metodologia contextualizada sem exclusão das evidências relacionadas (Souza et al., 2010).

Para nortear o desenvolvimento da pesquisa utilizou a pergunta norteadora: "De acordo com achados da literatura, qual a importância do clampeamento tardio do cordão umbilical, e os possíveis benefícios de tal prática para os Recém Nascidos $(\mathrm{RN})$ ?".

A busca foi realizada nas seguintes bases de dados: Literatura Latino Americana e do Caribe em Ciências da Saúde (LILACS); Base de Dados em Enfermagem (BDENF); National Library of Medicine, Sistema Online de Busca e Análise de Literatura Médica (Medline); Scientific Electronic Library Online (SciELO).

Adicionalmente, foram incluídos artigos completos e que estavam disponíveis de maneira gratuita eletronicamente em inglês, espanhol e português dos anos de 2016 a 2020 que mantinha-se dentro do tema proposto. Os critérios de exclusão serão artigos repetidos e que estavam fora do tema proposto, como também os incompletos.

Para operacionalização da pesquisa, foi utilizada a terminologia em saúde (DeCS), através dos quais se identificaram os descritores: "Parto Humanizado", "Cordão Umbilical", "Enfermagem Obstétrica", juntamente com o operador booleano "AND" e os descritores em inglês utilizamos o MeSH "Fetal Blood", "Umbilical Cord", "Obstetric Nursing".

Foram identificados 6.914 artigos nas bases de dados citadas anteriormente. Visto que, na etapa da triagem, foram excluídos os artigos repetidos e que estavam fora do tema proposto, como também os incompletos restando 4.303 artigos. Porém, após passarem pela etapa de elegibilidade, no qual foi realizada uma leitura restaram 540 artigos.

Após uma filtragem e demarcação dos critérios de exclusão foram selecionados como artigos finais para elaboração da presente pesquisa apenas 6 artigos, sendo que 1 artigos na base de dados do LILACS, 1 no BDENF, 1 no SCIELO e 3 no Medline. 
Figura 1: Apresentação do fluxograma com os artigos selecionados para a construção dessa revisão.
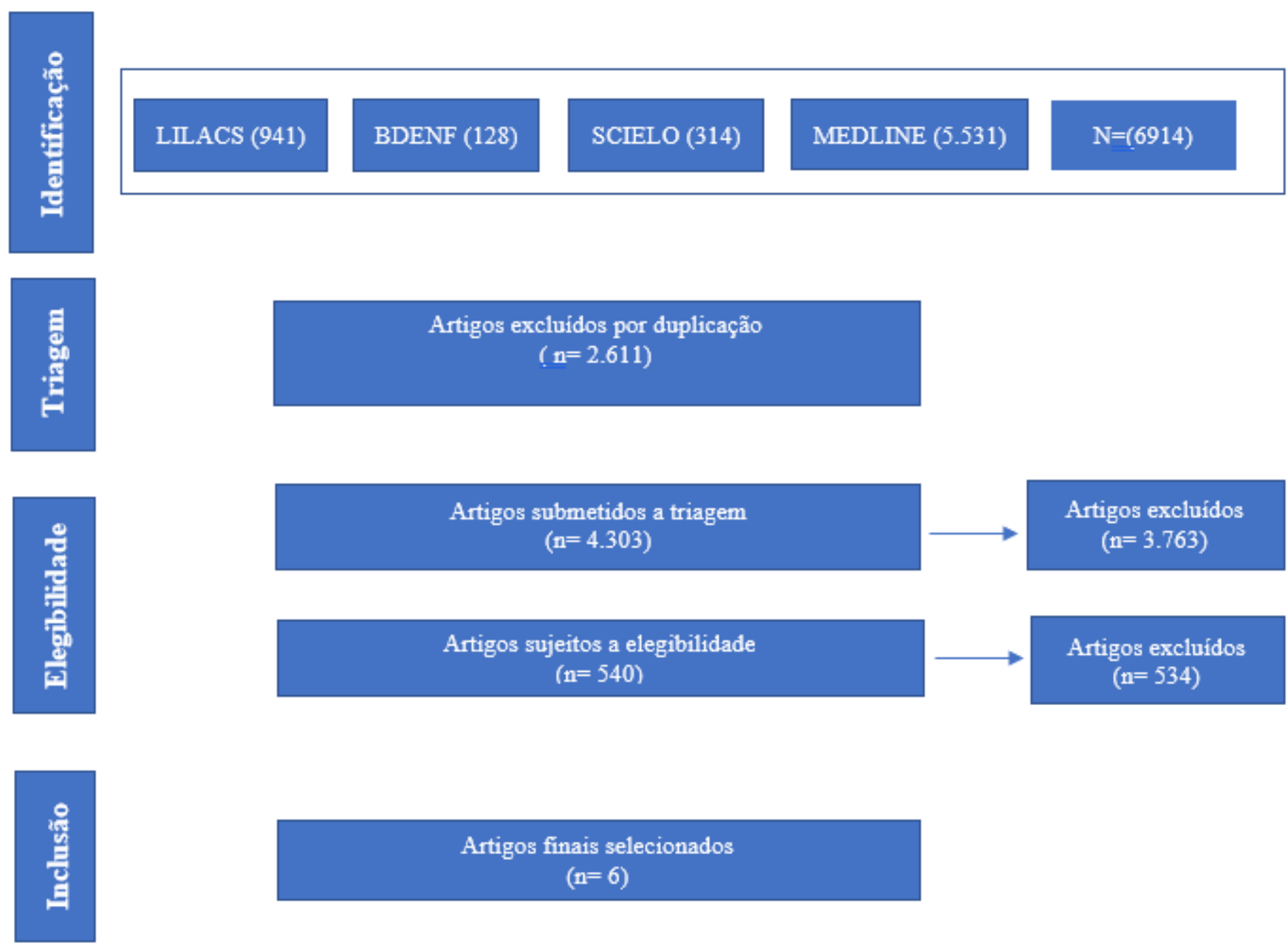

Fonte: Autores (2020).

Desse modo, apresentaram-se através de tópicos, considerando os aspectos encontrados de acordo com as temáticas abordadas pelo estudo. Os resultados foram feitos juntamente com a discursão que apresenta como objetivo a interpretação dos resultados com a descrição dos achados da literatura, explicando um novo entendimento sobre o assunto com base nos novos resultados.

\section{Resultados}

Na tabela abaixo estão delineados os 6 artigos finais para a construção dessa revisão. Foram organizados e apresentados com seus referentes autores, título do artigo, ano, objetivo e os principais resultados. Trata-se de uma sinopse dos resultados com maior destaque para este estudo, nos quais permanecem na íntegra da assistência de enfermagem obstétrica, boas práticas assistenciais ao recém-nascido, capacitação dos profissionais com boas práticas na assistência, as vantagens do clampeamento tardio, a coleta correta do cordão umbilical e o sangue do cordão umbilical para tratamento de doenças autoimunes e infecciosas. 
Quadro 1: Apresentação e principais resultados dos artigos eleitos para a pesquisa.

\begin{tabular}{|c|c|c|c|c|}
\hline $\begin{array}{l}\text { Lopes }^{1} \\
\text { Bernandino }^{2} \\
\text { Crozeta }^{3} \text { Guimarães }\end{array}$ & $\begin{array}{l}\text { Boas práticas para a coleta de } \\
\text { sangue do cordão umbilical e } \\
\text { placentário }\end{array}$ & 2016 & $\begin{array}{l}\text { Descrever os fatores } \\
\text { relacionados a qualidade das } \\
\text { amostras com boas práticas } \\
\text { durante a coleta de sangue do } \\
\text { cordão e placentário em um } \\
\text { banco púbico de sangue do } \\
\text { cordão umbilical. }\end{array}$ & $\begin{array}{l}\text { Analisados por meio de uma escala } \\
\text { onde foi possível observar as condutas } \\
\text { realizadas pelos enfermeiros e a equipe } \\
\text { obstétricas envolvidas no processo da } \\
\text { coleta do sangue. Diante das condutas } \\
\text { praticadas pelos profissionais foi } \\
\text { possível aplicar boas práticas como } \\
\text { ferramentas gerenciais para o trabalho } \\
\text { dos enfermeiros, com o objetivo de } \\
\text { obter amostras sanguíneas de alta } \\
\text { qualidade. }\end{array}$ \\
\hline $\begin{array}{l}\text { Oliveira }{ }^{1} \text { Paula }^{2} \\
\text { Garcia }^{3} \quad \text { Andrade }^{4} \\
\text { Leite }^{5}\end{array}$ & $\begin{array}{l}\text { Assistência obstétrica no } \\
\text { processo de parto e nascimento }\end{array}$ & 2018 & $\begin{array}{l}\text { Caracterizar a assistência } \\
\text { obstétrica no Brasil durante o } \\
\text { processo de parto e } \\
\text { nascimento, vivenciadas } \\
\text { pelos docentes de uma } \\
\text { Universidade Pública de } \\
\text { Minas Gerais. }\end{array}$ & $\begin{array}{l}\text { Coleta de dados com } 40 \text { docentes da } \\
\text { área nos anos de } 2015 \text { a } 2016 \text {, onde as } \\
\text { informações foram coletadas por meio } \\
\text { de questionamento autoaplicável e para } \\
\text { análise de dados utilizaram o teste de } \\
\text { Mann- Whitney. }\end{array}$ \\
\hline $\begin{array}{lc}\text { Narchi }^{1} & \text { Venâncio }^{2} \\
\text { Ferreira }^{3} & \text { Vieira }^{4}\end{array}$ & $\begin{array}{l}\text { O plano individual de parto } \\
\text { como estratégia de ensino- } \\
\text { aprendizagem das boas } \\
\text { práticas de atenção obstétrica }\end{array}$ & 2019 & $\begin{array}{l}\text { Verificar o conhecimento } \\
\text { dos alunos sobre o } \\
\text { planejamento individual do } \\
\text { parto e abordar opiniões } \\
\text { diante da estratégia de } \\
\text { ensino-aprendizado para } \\
\text { boas condutas. }\end{array}$ & $\begin{array}{l}\text { Os alunos foram entrevistados atrás de } \\
\text { um formulário, onde eles relataram } \\
\text { sobre o planejamento no pré-natal e o } \\
\text { cuidado prestado durante a assistência } \\
\text { ao parto, visando priorizar o } \\
\text { protagonismo da mulher. }\end{array}$ \\
\hline $\begin{array}{lr}\text { Basile }^{1} & \text { Pinelli }^{2} \\
\text { Micelli }^{3} & \text { Caretto }^{4} \\
\text { Panici }^{5} & \end{array}$ & $\begin{array}{l}\text { Ordenha do cordão umbilical } \\
\text { em bebês nascidos a termo e } \\
\text { prematuros tardios }\end{array}$ & 2019 & $\begin{array}{l}\text { Descrever a importância da } \\
\text { ordenha correta na secção } \\
\text { tardia do cordão umbilical e } \\
\text { seus benefícios durante esse } \\
\text { processo. }\end{array}$ & $\begin{array}{l}\text { Através de um analise nos estudos } \\
\text { selecionados pelo artigo. Observaram } \\
\text { que o corte do cordão precoce, priva os } \\
\text { volumes sanguíneos da placenta que } \\
\text { retorna para a criança durante o } \\
\text { clampeamento tardio. }\end{array}$ \\
\hline 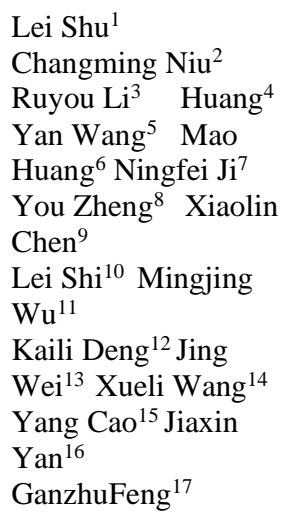 & $\begin{array}{l}\text { Tratamento de COVID-19 } \\
\text { grave com células-tronco } \\
\text { mesenquimais de cordão } \\
\text { umbilical humano }\end{array}$ & 2020 & $\begin{array}{l}\text { Descrever a eficácia da } \\
\text { Infusão de células troco- } \\
\text { mesenquimais como uma } \\
\text { opção de tratamento de } \\
\text { covid-19 de forma grave. }\end{array}$ & $\begin{array}{l}\text { Realizou um estudo em pacientes com } \\
\text { covid-19 grave. O tratamento com as } \\
\text { células tronco mesenquimais, foram } \\
\text { administradas por meio de transplante } \\
\text { intravenoso, obtendo-se resultados } \\
\text { positivos e eficazes como uma boa } \\
\text { opção que auxilia no tratamento de } \\
\text { pacientes com covid-19. }\end{array}$ \\
\hline $\begin{array}{l}\text { Ledo }^{1} \quad \text { Góes }^{2} \\
\text { Santos }^{3} \\
\text { Ávila }^{4} \\
\text { Silva }^{5}\end{array}$ & $\begin{array}{l}\text { Fatores associados às práticas } \\
\text { assistenciais ao recém-nascido } \\
\text { na ala de parto }\end{array}$ & 2020 & $\begin{array}{l}\text { Analisar os fatores que } \\
\text { influenciam em boas } \\
\text { práticas assistenciais ao } \\
\text { recém-nascido no momento } \\
\text { do parto. }\end{array}$ & $\begin{array}{l}\text { Realizado coleta de dados em } \\
\text { prontuários de nascimento entre os anos } \\
\text { de } 2015 \text { a } 2017 \text {, em conjunto com o } \\
\text { teste de Qui-Quadrado e a regressão } \\
\text { holística. Com o objetivo de coletar as } \\
\text { práticas realizadas na sala de parto } \\
\text { pelos profissionais. }\end{array}$ \\
\hline
\end{tabular}


O autor Ledo abordou em seus estudos sobre as boas práticas assistências ao RN na sala de parto, porém o autor Oliveira, realizou uma abordagem sobre a assistência cedida as puérperas no brasil durante o trabalho de parto. Os autores tiveram a mesma linha de raciocino sobre a assistência ofertada durante o trabalho de parto á puérpera e ao RN. O autor Narchi teve a mesma abordagem cientifica dos autores Ledo e Oliveira, onde ele destacou o plano de cuidados ao parto como uma forma de estratégia de ensino- aprendizado em boas práticas obstétricas.

Sendo assim, O autor Lopes descreveu sobre a qualidade das amostras coletadas do sangue do cordão umbilical, enfatizando as boas práticas durante a coleta para serem armazenadas de forma correta e encaminhados aos bancos públicos. O autor Lei Shu, trouxe um estudo recente sobre a utilização das Células-tronco Mesenquimais como uma forma de tratamento promissor para a Covid-19 e que apresenta resultados eficazes nos testes. Por fim, o autor Basile apresenta a mesma proposta de Lopes, onde destaca a ordenha correta na secção tardia do cordão e seus possíveis benéficos durante a execução.

\section{Discussão}

Durante a transição do RN para a vida extrauterina existe uma série de alterações fisiológicas, tais como troca gasosa, equilíbrio ácido básico e atividade cardiovascular. No brasil a pratica do clampeamento tardio é escasso, devido á falta de conhecimento, qualificações dos profissionais e treinamento da equipe diante da assistência ofertada durante o nascimento. A prática do pinçamento tardio do cordão é uma conduta que faz parte do processo da assistência humanizada, que beneficia a criança no momento do parto e se estende até a primeira infância (Ledo et al., 2020; Oliveira et al., 2018).

É crucial que os profissionais tenham o conhecimento teórico e prático de que a secção tardia do cordão, permite o contato pele a pele da mãe com o bebê. Esse contato oportuniza que o bebê respire pelos pulmões como também oportuniza o recebimento de oxigênio do cordão umbilical (Narchi et al., 2019).

A espera da secção tardia do cordão umbilical fornece uma melhora nos resultados neonatais, comparado a prática do clampeamento imediato. A secção tardia aumenta os níveis de transfusão placentária para o RN, permite que o volume sanguíneo retorne para o bebê cerca de $30 \%$ até $60 \%$ a mais no momento do nascimento. Esse aumento da transfusão placentária obtida pela pratica da secção tardia do cordão eleva os níveis de hemoglobina neonatal, conseguinte leva a um estoque de ferro que possibilita mais fluxo de hemácias para os órgãos vitais diminuindo o risco de anemia na infância (Basile et al., 2019).

Durante o acompanhamento dos bebês na internação hospitalar a transfusão da placenta e o clampeamento tardio do cordão demostraram vantagens ao bebê a termo com o aumento do volume de glóbulos vermelhos e volumes elevados de hematócritos, maior perfusão cutânea, temperatura da pele mais alta, acréscimo do fluxo sanguíneo renal com alta produção de urina e maior fluxo de glóbulos vermelhos para o cérebro e intestino (Basile et al., 2019).

De acordo com os estudos recentes, as Células-Tronco Mesenquimais do cordão umbilical (hUC-MSCs), estão sendo utilizadas no âmbito hospitalar contra doenças autoimunes e infecciosas que tem demostrado resultados positivos e segurança no tratamento da covid-19. As hUC-MSCs, mostrou eficácia e reparação imunológica significativa e reparação dos tecidos devido a sua baixa imunogenicidade. Além de desempenhar um papel protetor na lesão pulmonar que reduz a resposta inflamatória. Essas células quando acumuladas no pulmão podem melhorar o microambiente pulmonar, proteger as células epiteliais alveolares, evitar fibrose pulmonar e aprimorar a função pulmonar (Shu et al., 2020).

Além disso, os estudos destacam que essa terapia utilizando as (MSCP), também apresentou respostas positivas nos pacientes com complicações graves de diabetes, onde apresentaram menos uso de insulina exógena após a infusão das célulastronco mesenquimais. Com isto, esse tratamento pode ser indicado aos pacientes com diabetes e para os diabéticos com a covid19 grave. A terapia hUC-MSCs é um método experimental que está sendo testado, porém os resultados dos estudos analisados destacam que é um método eficaz e promissor como uma boa opção para ser utilizado como forma de tratamento, devido a ausência de medicamentos eficazes para o tratamento da covid-19 (Shu et al., 2020). 
É importante adoção de boas práticas e o conhecimento técnico e cientifica pela equipe de obstetrícia durante a coleta do sangue umbilical. Para obtenção de volumes e quantidades necessárias de células tronco aos bancos públicos de sangue do cordão umbilical e placentário, que são utilizados como tratamento por via transplante de pacientes com outras doenças malignas ou benignas (Lopes et al., 2016).

\section{Conclusão}

Diante desse estudo bibliográfico podemos observar que a secção tardia do cordão umbilical acarreta inúmeros benéficos para o RN durante o parto e na primeira infância, prevenindo doenças decorrentes nessa faixa etária. De acordo com os estudos abordados a espera ideal para realizar o clampeamento é de um a três minuto, visto que esse tempo não ocasiona riscos á saúde do bebê.

A espera da secção oportuniza o retorno do sangue circulante para o RN, beneficia os bancos públicos com o armazenamento de células-tronco que são utilizados para tratamento, oportuniza o contato pele a pele da mãe com o bebê que durante a espera permite que ele utilize o sistema respiratório devido o fornecimento de oxigênio do cordão umbilical e dentre outros privilégios.

É importante que os profissionais adotem mais essa prática durante o processo de parto, notamos que ainda há déficit por partes dos profissionais. Com isto, é relevante que as instituições aprimorem as capacitações e treinamentos das equipes ressaltando a relevância dessa prática que contribui para a saúde do bebê e oportuniza a humanização durante o trabalho de parto.

\section{Referências}

Basile, S. Pinelli, S. Micelli, E. Caretto, M. \& Panic, P. B. (2019). Ordenha do cordão umbilical em bebês nascidos a termo e prematuros tardios. Hindawi BioMed Research International. http://downloads.hindawi.com/journals/bmri/2019/9185059.pdf.

Brasil. (2017). Diretrizes Nacionais de Assistência ao parto normal. Brasilia: Ministério da Saúde http://bvsms.saude.gov.br/bvs/publicacoes/dire trizes_nacionais_assistencia_parto_normal.pdf.

Fionda, G. J. (2017). Clampeamento tardio do cordão umbilical: Estudo de corte [Dissertação]. Rio de Janeiro: Fundação Oswaldo Cruz Instituto Nacional de Saúde da Mulher, da Criança e do Adolescente Fernandes Figueira. https://www.arca.fiocruz.br/bitstream/icict/26357/2/juliana_goes_iff_mest_2017.pdf.

Leal, C. M. Bittencourt, A. S. Esteves- Pereira, A. P. Ayres, B. V. S. A. Silva, L. B. R. A. Thomaz, E. B. A. F. Lamy, Z. C. Nakamura-Pereira, M. Torres, J. Á. Nogueira da Gama, S. G. Domingues, R. M. S. M. \& Vilela, M. E. A. (2019). Avanços na assistência ao parto no Brasil: resultados preliminares de dois estudos avaliados. Cad. Saúde pública. 35(7), 1-14. https://www.scielo.br/pdf/csp/v35n7/1678-4464-csp-35-07-e00223018.pdf.

Ledo, B. C. Góes, F. G. B. Santos, A. S. T. Pereira-Ávila, F. M. V. Santana da Silva, A. C. S. \& Bastos, M. P. C. (2020). Fatores associados às práticas assistenciais ao recém-nascido na ala de parto. Esc Anna Nery. 25(1), e20200102. https://www.scielo.br/pdf/ean/v25n1/1414-8145-ean-25-1-e20200102.pdf

Lopes, L. A. Elizabeth, B. Karla, C. \& Paulo, R. B. G. (2016). Fatores associados às práticas assistenciais ao recém-nascido na ala de parto. Rev. Latino-Am. Enfermagem. 24, e2770. https://www.scielo.br/scielo.php?pid=S1414-81452021000100213\&script=sci_arttext.

Malheiros, G. C. \& Wilken \& de Abreu, A. M. O. (2016). Características morfológicas do cordão umbilical. Revista cientifica da FMC. 11(1), 1-5. http://www.fmc.br/ojs/index.php/RCFMC/article/view/8/7.

Narchi, N. Z. Venâncio, K. C. M. Ferreira, F. M. \& Vieira, J. R. (2019). O plano individual de parto como estratégia de ensino-aprendizagem das boas práticas de atenção obstétrica. Rev Esc Enferm USP. 53, e03518. https://www.scielo.br/pdf/reeusp/v53/1980-220X-reeusp-53-e03518.pdf

Oliveira, J. C. Paula, A. C. S. Félix Garcia, E. S. G. Tinti de Andrade, M. B. \& Carvalho Leite, E. P. R. (2018). Assistência obstétrica no processo de parto e nascimento. J. res: fundam. Cuidado. Online. 10(2), 450-457. file:///C:/Users/Fabrica/Downloads/bdenf\%201ilasc\%20enfermagem\%20obestrtrica\%20(1).pdf.

Shu, L. Changming, N. Ruyou, L. Tingrong, H. Yan, W. Mao, H. Ningfei, J. Você, Z. Xiaolin, C. Lei, S. Mingjing, W. Kaili, D. Jing, W. Xueli, W. Yang, C. Jiaxin, Y. \& Ganzhu, F. (2020). Tratamento de COVID-19 grave com células-tronco mesenquimais de cordão umbilical humano. Pesquisa e terapia com célulastronco. 21, 361. https://stemcellres.biomedcentral.com/articles/10.1186/s13287-020-01875-5.

Vain, N. E. (2015). Em tempo: como e quando deve ser feito o clampeamento do cordão umbilical: será que realmente importa? Elsevier Editora Ltd. 33(3), 12. https://www.scielo.br/scielo.php?script=sci_arttext\&pid=S010305822015000300258\#: :text=Com\%20base $\% 20 \mathrm{em} \% 20 \mathrm{ensaios} \% 20 \mathrm{cl} \% \mathrm{C} 3 \% \mathrm{~A}$ Dnico s,b eb\% C3\%AAs\%20aumenta\%20uma\%20m\%C3\%A9dia\%20101. 